\title{
Lumbar Vertebral Fracture L2
}

National Cancer Institute

\section{Source}

National Cancer Institute. Lumbar Vertebral Fracture L2. NCI Thesaurus. Code C27336.

A traumatic break in the second lumbar spinal vertebra. 